\title{
A two-dimensional computational parametric analysis of the sheltering effect of fences on a railway vehicle standing on a bridge and experiencing crosswinds
}

\author{
Ignacio Sesmal,2, Gorka S. Larraona2, Jordi Vinolas ${ }^{1,2}$, \\ Alejandro Rivas' and Sergio Avila-Sanchez ${ }^{3}$
}

\begin{abstract}
In a crosswind scenario, the risk of high-speed trains overturning increases when they run on viaducts since the aerodynamic loads are higher than on the ground. In order to increase safety, vehicles are sheltered by fences that are installed on the viaduct to reduce the loads experienced by the train. Windbreaks can be designed to have different heights, and with or without eaves on the top. In this paper, a parametric study with a total of 12 fence designs was carried out using a two-dimensional model of a train standing on a viaduct. To asses the relative effectiveness of sheltering devices, tests were done in a wind tunnel with a scaled model at a Reynolds number of $\mathrm{I} \times 10^{5}$, and the train's aerodynamic coefficients were measured. Experimental results were compared with those predicted by Unsteady Reynoldsaveraged Navier-Stokes (URANS) simulations of flow, showing that a computational model is able to satisfactorily predict the trend of the aerodynamic coefficients. In a second set of tests, the Reynolds number was increased to $12 \times 10^{6}$ (at a free flow air velocity of $30 \mathrm{~m} / \mathrm{s}$ ) in order to simulate strong wind conditions. The aerodynamic coefficients showed a similar trend for both Reynolds numbers; however, their numerical value changed enough to indicate that simulations at the lower Reynolds number do not provide all required information. Furthermore, the variation of coefficients in the simulations allowed an explanation of how fences modified the flow around the vehicle to be proposed. This made it clear why increasing fence height reduced all the coefficients but adding an eave had an effect mainly on the lift force coefficient. Finally, by analysing the time signals it was possible to clarify the influence of the Reynolds number on the peak-to-peak amplitude, the time period and the Strouhal number.
\end{abstract}

\section{Keywords}

Train aerodynamics, train overturning, overturning risk, computational fluid dynamics, Unsteady Reynolds-averaged Navier-Stokes, bluff bodies, URANS

\section{Introduction}

The effect of crosswinds over trains has become an important topic in recent years as train velocities have increased and the tendency of designers has been to lighten vehicles. Because it is a matter of safety, the topic is relevant to the scientific community and subject to study. The goal of increasing velocity is to make rail transportation competitive against other means of transport; therefore, it is vital that the problem is addressed and solutions are proposed.

High-speed lines are designed to minimize curves: they are built with as many kilometres of straight stretches as possible, and when turnouts are needed, the radius of the curve is maximized. This means that a large number of bridges have to be constructed in order to cross valleys, and many embankments are needed to make the terrain on which the rail is built uniform. It is widely known that wind velocity increases as the distance to the surface increases; thus, under the same wind conditions, a train will have to withstand higher wind loads on a bridge than on flat terrain. In order to protect the vehicle

\footnotetext{
'CEIT, San Sebastián, Spain

${ }^{2}$ Tecnun, University of Navarra, San Sebastián, Spain

${ }^{3}$ Universidad Politécnica de Madrid, Madrid, Spain

Corresponding author:

Ignacio Sesma, CEIT, Manuel de Lardizábal 15, 20018 San Sebastián, Spain

Email: isesma@ceit.es
} 
so it can run without reducing velocity, windbreaks, such as fences, are currently being installed on bridges and other locations which are exposed to the wind. These fences reduce wind loads and protect the train from the wind by redirecting most of the flow over the vehicle, thus making its operation safer. There are several kinds of windbreaks: solid or porous, with different heights and with or without eaves. All these configurations have the same goal, to protect the train, but each of them does it by modifying the wind flow in a different way.

The research community at the TRANSAERO project ${ }^{1}$ contributed to the study of windbreaks by performing computational fluid dynamics (CFD) simulations, wind tunnel experiments and on-track tests. Most of the project's work focused on tracks built on embankments and it was a breakthrough in the state-of-the-art of the topic. However, more research needs to be undertaken, mainly on the problem of running trains over bridges. Several studies based on experimental tests in wind tunnels ${ }^{25}$ have looked at the behaviour of the air flow when it encounters a bridge that does not have windbreaks, studying the effect of the Reynolds number on the calculation of the aerodynamic coefficients and on the vortex shedding phenomenon that appears on the leeward side of bridges. A CFD model of a bridge was used by Mannini et al. ${ }^{6}$ in Unsteady Reynolds-averaged Navier-Stokes (URANS) simulations that were performed to validate an experiment. Wind tunnel tests on a two-dimensional model of a bridge were performed by Avila-Sanchez et al. ${ }^{7}$ to study how fences influence the turbulence intensity at the catenary contact wires. However, only a few studies focus on the vehicle. Bettle et al. ${ }^{8}$ analysed the problem of a trailer on a bridge using threedimensional CFD simulations. Diedrichs ${ }^{9,10}$ performed CFD computations and discussed in detail the aerodynamics of high-speed trains. Cheli et al. ${ }^{11}$ used wind tunnel tests to prove that for a Reynolds number $(R e)$ up to $1 \times 10^{6}$, force coefficients are slightly sensitive to changes in $R e$ for the case of a train running on a track equipped with fences. Barcala and Meseguer ${ }^{12}$ studied the effect of parapets on the aerodynamic loads experienced by a vehicle on a bridge using two-dimensional tests carried out in a wind tunnel. They showed that fences are able to significantly reduce the wind loads that the vehicle withstands.

When designing a new wind fence, the influence of parameters such as the height and length of the eaves, if there are any, needs to be tested in order to decide which designs are the most appropriate to safeguard the train from the wind in a particular scenario. Tests can be done in a wind tunnel since the literature proves it to be a valid method; however, the costs of running these experiments are high and using a wind tunnel may also not be the most effective approach since each study requires a particular mock-up be built. In addition, the Reynolds number that can be achieved in experimental tests is limited by the size and the power of windtunnels, which makes it almost impossible to represent real wind conditions. In recent years, CFD has appeared as a suitable alternative to wind tunnel tests because it is able to simulate flow accurately and allows real wind conditions to be reproduced.

This paper analyses the relative effectiveness of solid wind fences that are built on a bridge to decrease the aerodynamic loads that might result in a vehicle overturning in strong wind conditions. In the scope of a two-dimensional study, this work discusses the design parameters of fences, i.e. fence height and eave length, along with how such fences modify the flow structure since fences determine the difference in pressure on the surface of the vehicle and thus the aerodynamic coefficients. This methodology is shown to be valid and it can already be found in the literature, as in, Barcala and Meseguer ${ }^{12}$ and Sanz et al. ${ }^{13}$

The analysis will be made from the point of view of whether the vehicle is more protected and whether loads over it are lower. The method applied herein makes it possible to disregard any fence geometry that does not result in a significant reduction of the loads on the bluff body. However, this study does not discuss how fences modify the wake around a highspeed train since a three-dimensional study is required for that purpose.

Tests at a Reynolds number $R e \approx 1 \times 10^{5}$ were performed in a wind tunnel to check the computational model. Results show that the CFD model satisfactorily reproduces the trends of the coefficients that were measured in wind tunnel tests. Simulations were performed again increasing the Reynolds number to $R e \approx 12 \times 10^{6}$ to simulate strong wind conditions. The problem must be studied using URANS equations because there is a vortex shedding phenomenon that forces the study to go beyond steady state simulations. The influence of the fence design on the frequency and peak amplitude of the time histories of the aerodynamic coefficients is also studied.

Finally, by comparing the simulations at both Reynolds numbers it is possible to analyse the trend and the numerical value of the aerodynamic coefficients taken at two different wind velocities.

\section{Problem description and definitions CFD model}

Geometry, flow domain and boundary conditions. The high-speed vehicle used in this study was the RENFE Class 120 from the Spanish train manufacturer CAF. After being pre-processed in CAD software, the real cross-sectional shape of a passenger coach and a typical section of a bridge were introduced into the computational model in real scale (see Figure 1). 
Since the aim of this paper is to analyse the influence of solid fences, it was necessary to parameterize them by height, $h_{\mathrm{f}}$, and the length of the eave, $l_{\mathrm{e}}$. Four fence heights were tested: $h_{\mathrm{f}}=1250,1750,2250$ and $2750 \mathrm{~mm}$. Three eave lengths were also considered: no eave, $l_{\mathrm{e}}=500$ and $750 \mathrm{~mm}$. In addition to the scenario where no fence is installed, a total of 12 different fence designs were considered.

The train was located at the critical side, i.e. the windward side, of a double-track bridge since that is the side where the train is most exposed to wind. At the leeward side, the shielding effect of the fences increases and so wind loads over the vehicle are lower. $^{12}$

The aerodynamic loads per unit length, drag force $(D)$, lift force $(L)$ and roll moment $\left(M_{0}\right)$ are calculated at the centre of the carbody (see Figure 1). $M_{v}$ is the moment around the leeward rail, which measures the turnover of the vehicle caused by crosswinds loads. It assesses the efficiency of the parapets against overturning in a single coefficient since it takes into account the contribution of all the aerodynamic loads. The vehicle will be at risk of overturning when the restoring moment due to the vehicle's mass does not keep the vehicle in equilibrium.

In this paper, the dimensionless aerodynamic force coefficients, $C_{d}$ and $C_{l}$, and the moment coefficient, $C_{m 0}$, are calculated according to standard fluid dynamics formulas. For these coefficients, the train's width $(W)$ and height $(H)$ are used

$$
\begin{aligned}
C_{d} & =\frac{D}{0.5 \rho U_{\infty}^{2} H} \\
C_{l} & =\frac{L}{0.5 \rho U_{\infty}^{2} H} \\
C_{m_{0}} & =\frac{M_{0}}{0.5 \rho U_{\infty}^{2} H W}
\end{aligned}
$$

where $U_{\infty}$ is the free flow air velocity and $\rho$ the air density.

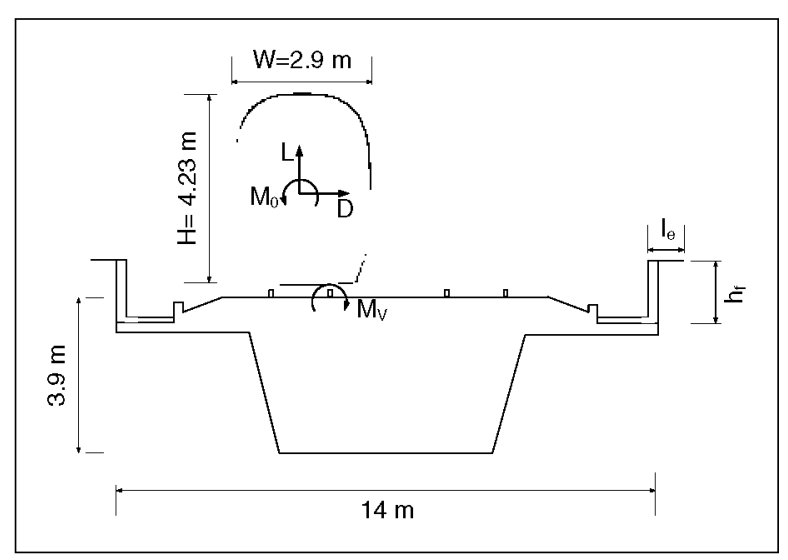

Figure I. Main vehicle and bridge dimensions in the CFD model. Definition of fence height $\left(h_{f}\right)$, eave length $\left(l_{e}\right)$, carbody height $(H)$ and carbody width $(W)$.
The Reynolds number $\left(\operatorname{Re}=\left(U_{\infty} \times L_{\mathrm{c}}\right) / \nu\right)$ was calculated with the kinematic viscosity of the fluid, $v$, (see the section 'Flow assumptions, turbulence model and numerical considerations') and by considering as the characteristic length, $L_{\mathrm{c}}$, the distance from the top of the coach to the bridge base $(8.5 \mathrm{~m})$. The Strouhal number, which is a dimensionless number that describes the oscillations of the flow, was used to analyse the vortex shedding. It is defined as $S t=\left(f \times L_{\mathrm{c}}\right) /$ $U_{\infty}$, where $f$ is the frequency of the vortex shedding.

$C_{m v}$ is determined by calculating a simple force and moment equilibrium at the position of the leeward rail

$$
C_{m_{v}}=\frac{1}{2} C_{d} \frac{H}{W}+\frac{1}{2} C_{l} \frac{b_{0}}{W}-C_{m 0}
$$

where $b_{0}$ is the distance between contact points $(1.5 \mathrm{~m})$. An analysis of the equation shows that the effect of the roll moment is relatively small.

The computational domain was two-dimensional and represented by a rectangle (Figure 2). To reproduce wind tunnel tests, a uniform profile was imposed at the inlet boundary. Depending on the simulation type, the velocity value can be $0.22 \mathrm{~m} / \mathrm{s}\left(R e \approx 1 \times 10^{5}\right)$ to reproduce wind tunnel tests or $30 \mathrm{~m} / \mathrm{s}$ ( $R e \approx 12 \times 10^{6}$ ) to represent strong wind conditions. The turbulence intensity was $3 \%$ and the turbulence viscosity ratio had a value of five. The latter is the ratio between the turbulent viscosity, which is a property of the flow, and the viscosity of the fluid. At the outlet, a constant pressure boundary was adopted. Finally, for the top and bottom faces a symmetry condition was selected (see Figure 2). The external borders of the domain are far enough apart to simulate the free flow condition. The blockage factor of the computational domain was 0.04 , which ensured that the distortion of the flow was reduced to a minimum.

The two-dimensional approach used in this paper is valid for fulfilling the objective of this paper, which is to compare different wind fence designs and to determine which features (fence height and eave length) are more important from a safety perspective, by comparing fences to each other. Three-dimensional calculations provide the best approach to real

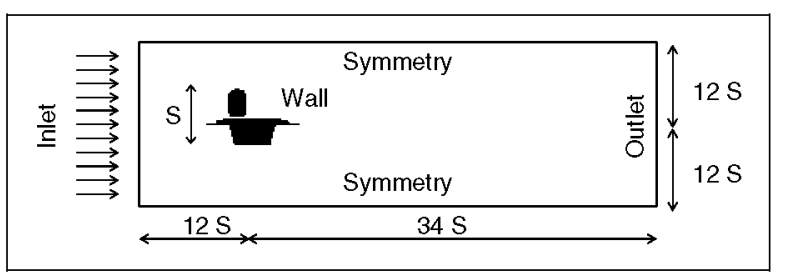

Figure 2. Non-scaled representation of the CFD domain. Dimensions are given in terms of the height of the cross-section of the bridge plus the vehicle (S). The boundary conditions are in grey. 
phenomena since they are able to represent the wake formed by the train's nose, which determines behaviour against crosswinds. However, they are not the most efficient way if one wants to complete a parametric study. These kinds of calculations require extensive computational resources and they are costly in most cases because of the time it takes to obtain a set of results.

Two-dimensional simulations are a useful tool for comparing the relative effectiveness of the wind fences and for measuring the efficiency of train sheltering. When there is a set of wind fences and one wants to select a few from among the set in order to carry out a detailed study in three dimensions, two-dimensional simulations are sufficient.

Flow domain discretization. The flow domain (see Figure 2) was meshed with quadrilateral elements, ranging from $1.5 \times 10^{6}$ elements in the no-fence scenario to $2.5 \times 10^{6}$ elements in the scenarios where fences with eaves were installed. The mesh is non-uniform, meaning that the element size grows as the cell gets further from the area surrounding the vehicle and bridge. Mesh quality was measured in terms of a value (equiangle skew) which measures their angular distortion (zero: no distorted and one: highly distorted). The equiangle skew average of the whole domain was near zero, i.e. $1 \times 10^{-5}$. In the area surrounding the vehicle and bridge, more than $99 \%$ of the elements in that area had an equiangle skew below 0.3 .

A layer of fine cells in the vicinity of the carbody was included. The first element of this layer was $0.25 \mathrm{~mm}$ in height to accurately solve velocity gradients and to keep the non-dimensional distance from the wall, $y+$, below 30 at the highest Reynolds number. No layers were included around the bridge because this study was not focused on the analysis of the wind loads on the bridge, but elements were kept sufficiently small to represent the flow accurately.

To make sure that the results were independent of the mesh, the scenario in which the vehicle stands on the bridge without any fence was meshed again. The refinement ratio was 1.25 , resulting in a variation of the aerodynamic coefficients below $2 \%$ at both $R e$ values used in this study. After the refinement and the quality check, it was concluded that the mesh was good enough to carry out the study.

Flow assumptions, turbulence model and numerical considerations. The numerical calculations were made with the commercial CFD software package Ansys Fluent $^{14}$ with a pressure-based solver. The flow was assumed to be incompressible and isothermal. The air properties used were those for the standard conditions with a density of $1.225 \mathrm{~kg} / \mathrm{m}^{3}$ and a kinematic viscosity, $v$, of $1.7894 \times 10^{-5} \mathrm{~kg} /(\mathrm{m} \cdot \mathrm{s})$.

The air flow was considered to be turbulent, and the two-equation $k-\varepsilon$ turbulence model was chosen. The three versions of the model: Standard,
Realizable and RNG were tested with the scenario where the train is unsheltered. The obtained results showed that the Standard version fitted the data better than the others when they were contrasted with the tests carried out in the wind tunnel; therefore, this was the model that was used in the simulations.

In spite of having constant boundary conditions at the inlet, the nature of the problem is transient since when there is a bluff body inside an air stream, a vortex shedding phenomenon emerges. The convergence criterion was to reach the periodic regime, so the method consisted in obtaining the time histories of the coefficients by providing enough cycles so that this state was guaranteed. Thus, when the value of the aerodynamic coefficient is presented for a certain scenario, it is given as the average in time.

Each wind velocity value required a specific time step because the frequency of the vortex shedding phenomenon depends on $R e$; therefore, two time steps were needed. The step size was $1 \mathrm{~s}$ in the cases of $U_{\infty}=0.22 \mathrm{~m} / \mathrm{s}$, whereas for $U_{\infty}=30 \mathrm{~m} / \mathrm{s}$ it was $0.02 \mathrm{~s}$; this made it possible to drive the scaled residuals to $10^{-6}$ in each time step.

The pressure/velocity coupling was made in terms of the PISO algorithm. ${ }^{15}$ Second-order upwind numerical schemes were employed for the convective terms of momentum and turbulence magnitude equations. $^{16}$

\section{Experimental model and setup}

Experimental tests in a wind tunnel were carried out by IDR/UPM, E.T.S.I. Aeronáuticos, Universidad Politécnica de Madrid with the aim of checking the CFD model. The experimental setup sought to characterize the aerodynamic loads on the coach's surface in order to calculate the aerodynamic coefficients that could be used to make a comparison with the results of the CFD model. This means that no conclusion was extracted from the test campaign other than testing the CFD model. In order to fulfill this goal, an open-circuit wind tunnel with a closed test section was used to perform a set of two-dimensional tests. The wind tunnel's working section is $1.8 \mathrm{~m}$ high, $0.2 \mathrm{~m}$ wide and $1.2 \mathrm{~m}$ long.

For the case study presented here and in order to reproduce the most important aerodynamic characteristics, a scale of $1 / 50$ was chosen for the whole mock-up. The geometry of the coach was simplified due to limitations of the mock-up manufacturing process. It will later be shown that this results in differences between the experimental and CFD results.

A Scanivalve Corp. pressure scanner, model ZOC33, with 128 pressure inputs, was used to measure the pressure on the model's surface. The train model was equipped with 48 evenly distributed pressure taps near the mid-section (Figure 3), each them connected to the pressure scanner through pneumatic inputs. A pitot tube located upstream from 


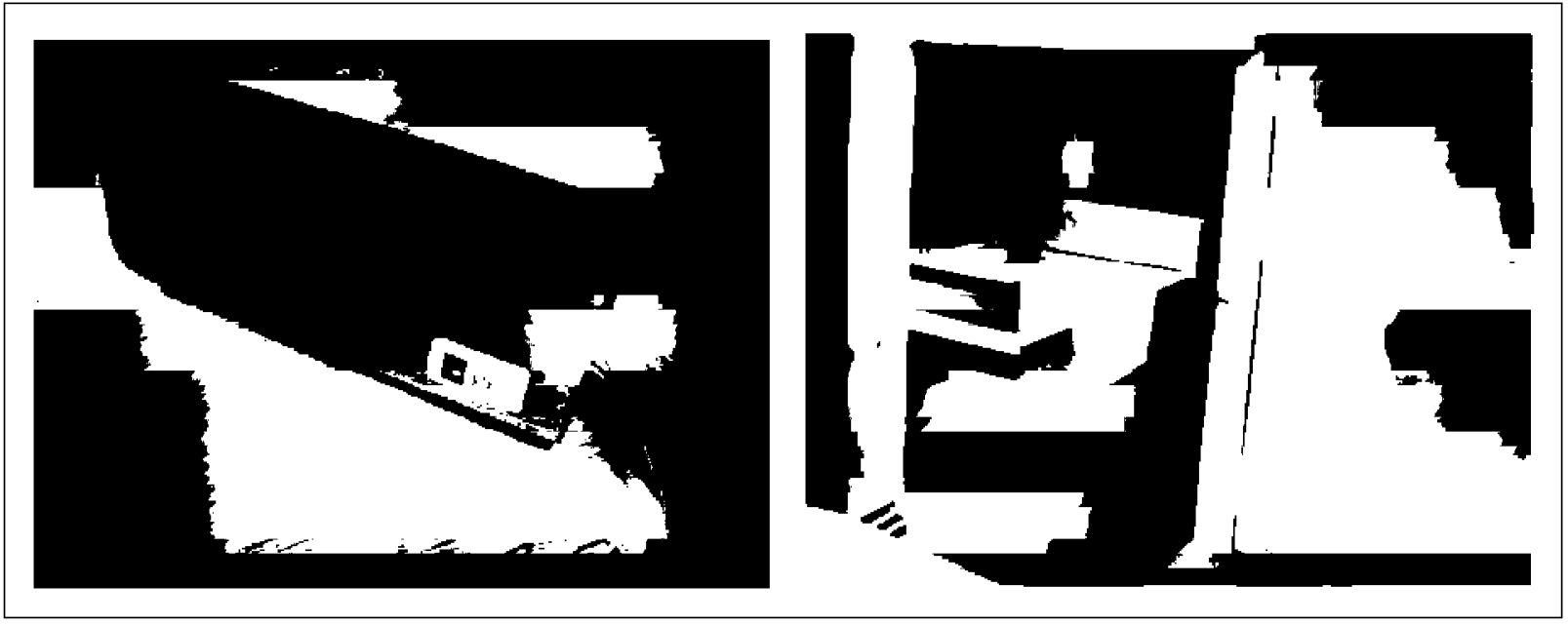

Figure 3. Mock-up of the vehicle and the bridge (left) and mock-up of the bridge with fences in the wind tunnel (right).

the mock-up and near the top of the chamber was used to measure both the total and static pressures to determine the dynamic pressure of the air flow. The time average value corresponding to each pressure tap was computed in order to determine the pressure coefficient. The global aerodynamic coefficients were obtained by numerical integration of the pressure coefficients on the model's surface. Different values for the sampling rate and the sampling period were checked to measure the pressure signal corresponding to each pressure tap. After several observations, the selected sampling period was taken to be $12 \mathrm{~s}$ since the average values of the coefficients did not change appreciably for larger sampling periods. The selected sampling rate was $100 \mathrm{~Hz}$ to fully capture the time history.

The Reynolds number at which the tests were performed was approximately $1 \times 10^{5}$ and the free flow speed was uniform and equal to $11 \mathrm{~m} / \mathrm{s}$ within $\pm 1 \%$. The free flow turbulence intensity was measured using a DANTEC (CTA module 90C10 and probe type 55P16) hot wire anemometer system. EN 1991 1-4, Eurocode $1^{17}$ gives recommendations for setting up the value of the free flow turbulence intensity, which is around $7 \%$ for the case of a location with a distance to the ground level that is similar to the case of a bridge. However, this standard was written for the study of the infrastructure and it does not cover the case of vehicles situated on it. Therefore, different values of turbulence intensity were checked in the range $3-10 \%$ by placing different grids at the beginning of the working section. Measurements showed that larger values of turbulence intensity produced slightly smaller loads on the vehicle. The reason for this effect is that wind tunnel tests with low turbulence levels create a more severe condition since the average of the experimental loads is higher. ${ }^{18}$ However, these differences are not significant since the bridge itself perturbs the flow enough to reduce the impact of this parameter. ${ }^{7}$ In this context, the selected value for the turbulence intensity was $3 \%$ since the safety margin is increased as actual loads are expected to be lower. No atmospheric boundary layer was reproduced since the height at which the vertical velocity gradient changes significantly is much larger than the height of the parapet.

Due to the limited dimensions of the test section, the blockage factor was close to 0.14 , which can be considered to be high. As a result of the blockage effect, the velocity of the airstream around the model increased slightly, which amplified the loads that the model withstood. Typical increases in the aerodynamic coefficients in this kind of experiment are expected to be $15 \%$, so the coefficients were corrected. Two-dimensional tests may require corrections due to the growth of a boundary layer in the tunnel as a consequence of the size of the mock-up and wake blockage caused by the model. ${ }^{19}$ The growth of boundary layers on the tunnel walls modifies the static pressure along the wind tunnel. For bodies whose longitudinal dimension is much larger than the vertical dimension, the effect is an increase in the aerodynamic resistance. Since the dimensions of the considered model are similar in both directions, this correction was not considered. Solid blockage corrections as well as wake corrections were applied based on the work of Barlow et al. ${ }^{20}$ However, corrections are based on empirical considerations, and thus the uncertainty in including the blockage effects can be considered an explanation for the disagreement between the experimental and computational values of the aerodynamic coefficients.

\section{Results}

The main goal of this section is to study the influence of fences and the wind flow around the vehicle. First, the CFD model was checked with the tests carried out in the wind tunnel at $R e \approx 1 \times 10^{5}$. Afterwards, the discussion presented here focuses on the fence design at $R e \approx 12 \times 10^{6}$ because the conditions it represents are closer to the real exposure of a train on a bridge 


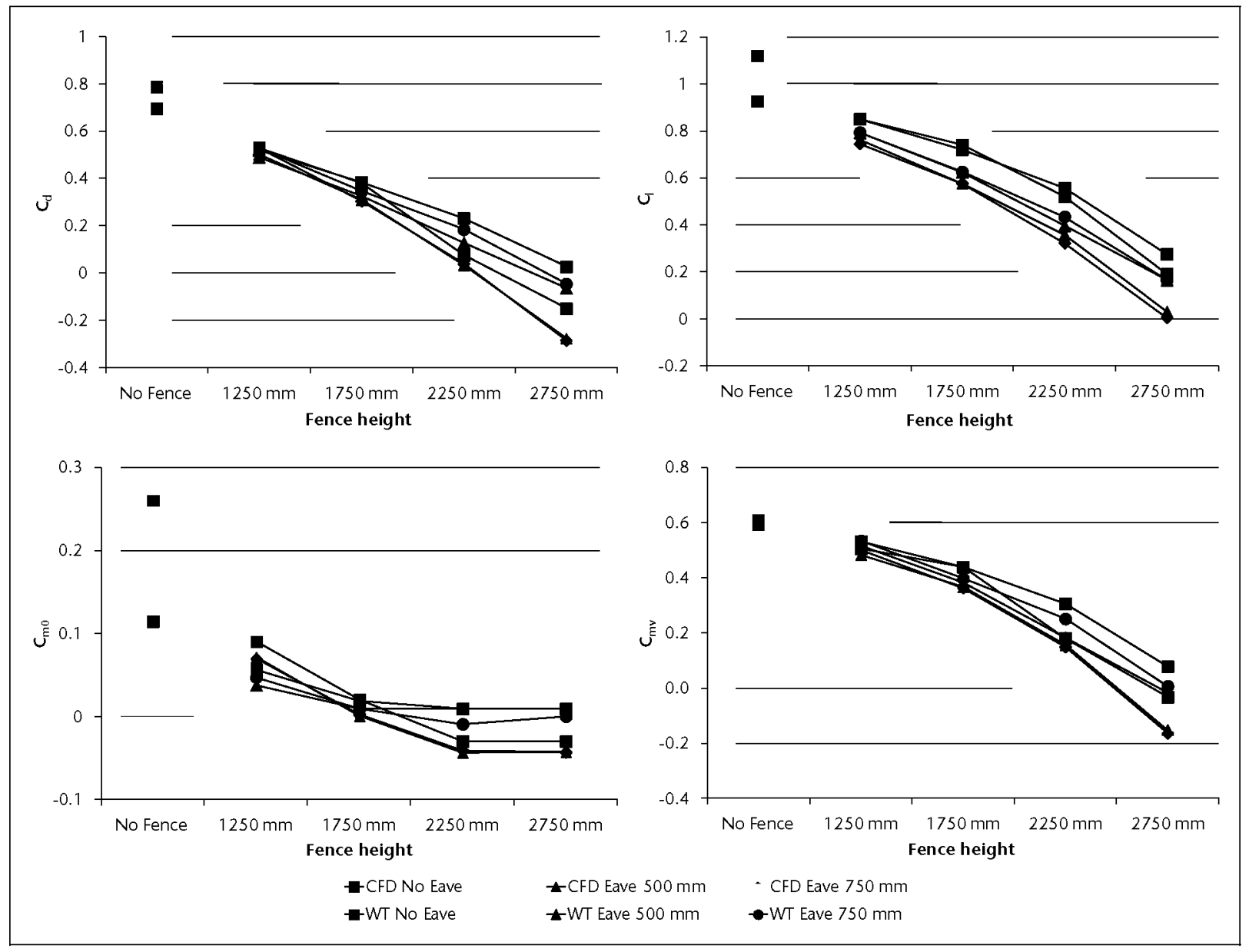

Figure 4. Comparison between the wind tunnel tests (WT) and computational results (CFD) for the given cases.

under strong crosswinds. However, it will be shown in the section 'Testing the CFD model' that the aerodynamic coefficients have the same trend with both $R e$ values. The transient regime of the problem was analysed by looking at the time signals of the coefficients calculated with the simulations at the highest Reynolds number. Then, the average values of the aerodynamic coefficients of the train coach were studied in order to find the most effective fence. At this point, conclusions regarding the fence construction parameters $\left(h_{\mathrm{f}}\right.$ and $l_{\mathrm{e}}$ ) were extracted. The flow around the vehicle and the bridge was observed in detail, establishing differences between the two wind velocities under consideration in this study. Finally, the vehicle coefficients from simulations with the two Reynolds numbers were compared, in order to determine whether Re influences the values of the coefficients.

\section{Testing the CFD model}

Wind tunnel tests were performed to check the CFD model and given the purpose of this paper only the mean values of the aerodynamic coefficients are provided.
According to the results presented in Figure 4, the CFD simulations satisfactorily predict the trends of the aerodynamic coefficients that were measured in wind tunnel tests. In general terms, the CFD model provides lower values for all the aerodynamic coefficients, the only exception being the case where the vehicle was not protected by a windbreak; in such a case CFD predicts higher values. There are three main causes that are expected to be sources of uncertainty: the turbulence model used in the CFD, the simplified geometry of the mock-up, and the wind tunnel blockage. The well-known limitations of the employed TRANS turbulence model introduce uncertainty in the CFD model for all the computed cases. The carbody of the mock-up does not exactly represent the true geometry of the vehicle but it does in the CFD model. The downside of the mock-up is flat whereas the true geometry has a low inclination angle. Moreover, the true lateral sides of the geometry are a succession of curves of different radii whereas the mock-up adopts the curves' envelope. This results in differences between the CFD model values and the experimentally obtained values for an unsheltered vehicle. A blockage can be the cause of obtaining larger differences between the computational model 


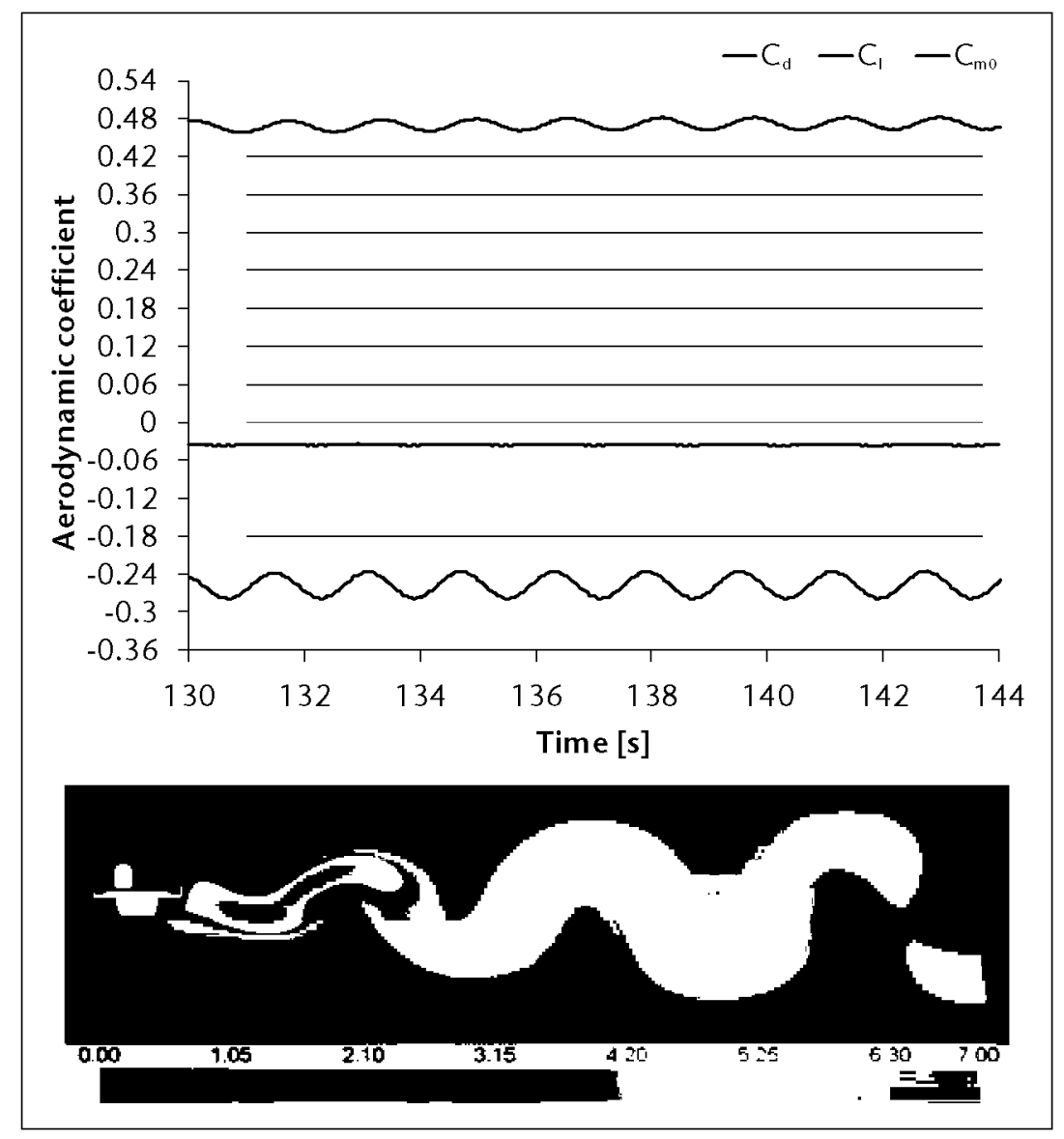

Figure 5. Time signals for the calculated coefficients for the case where $h_{\mathrm{f}}=2250 \mathrm{~mm}$ with an eave $750 \mathrm{~mm}$ (top) and a panoramic view of the scenario showing the turbulent viscosity $(\mathrm{kg} / \mathrm{m} \cdot \mathrm{s})$ (bottom). Calculations carried out at $\operatorname{Re} \approx 12 \times 10^{6}$.

and the experimental tests in the cases of $h_{\mathrm{f}}=2250 \mathrm{~mm}$ and $h_{\mathrm{f}}=2750 \mathrm{~mm}$.

Additionally, the free flow velocity is constant in CFD studies whereas in the wind tunnel it has variations of $\pm 1 \%$. The same applies to the turbulence intensity, where its value does not change in CFD but in tests it does so. However, the influence of the inlet conditions is expected to be low since the wind profile at the inlet is almost identical.

For the drag coefficient $\left(C_{d}\right)$, the difference between wind tunnel tests and simulations grows as the fence height increases. The computed coefficients of lift force $\left(C_{l}\right)$ and roll moment $\left(C_{m 0}\right)$ show the biggest disparity within the experimental tests on the configuration where the train was completely exposed to crosswinds. In the scenario where there is no fence to shelter the vehicle the coach reorients the flow, which makes it highly dependent on the carbody's geometry. Thus, as long as the geometry of the mock-up and the CFD model agree, the pressure differences between top and bottom agree and so do the aerodynamic coefficients of the lift and roll moment. Note that, in the case of $C_{m 0}$ the CFD model predicts reasonable well the effect of fences.

In view of the comparison, it appears that the CFD simulations are able to predict reasonably well the value of $C_{m v}$ extracted from the tests in the scenarios where the vehicle was unsheltered and when the fence height was 1250 and $1750 \mathrm{~mm}$. The difference increases in the case of the highest fence, which might be due to the larger effect of a blockage. $C_{m v}$ presents a similar trend to $C_{d}$, simulations accurately predict the situation of the unprotected vehicle, but the inaccuracy increases with fence height. Although $C_{m 0}$ also determines $C_{m v}$, its near-zero value when the bridge is equipped with fences make it insignificant.

It should be noted that having fences with eaves on top of them does not modify the presented conclusions. Simulations closely reproduce the difference between the geometry when the fence has no eave and the geometries when it does so. The computational model is in agreement with the experimental tests when adding an eave reduces the value of the coefficients but an increase in eave length has a very minor impact on the coefficients. In most cases, the CFD calculations predict smaller variations between the two eave lengths than the results obtained in the tests.

In general terms, CFD simulations are able to represent the variations in the aerodynamic coefficients as a result of building fences with eaves. The CFD model is able to reproduce the trends of the coefficients, showing the effect of increasing the fence height and also adding an eave. However, the difference between 


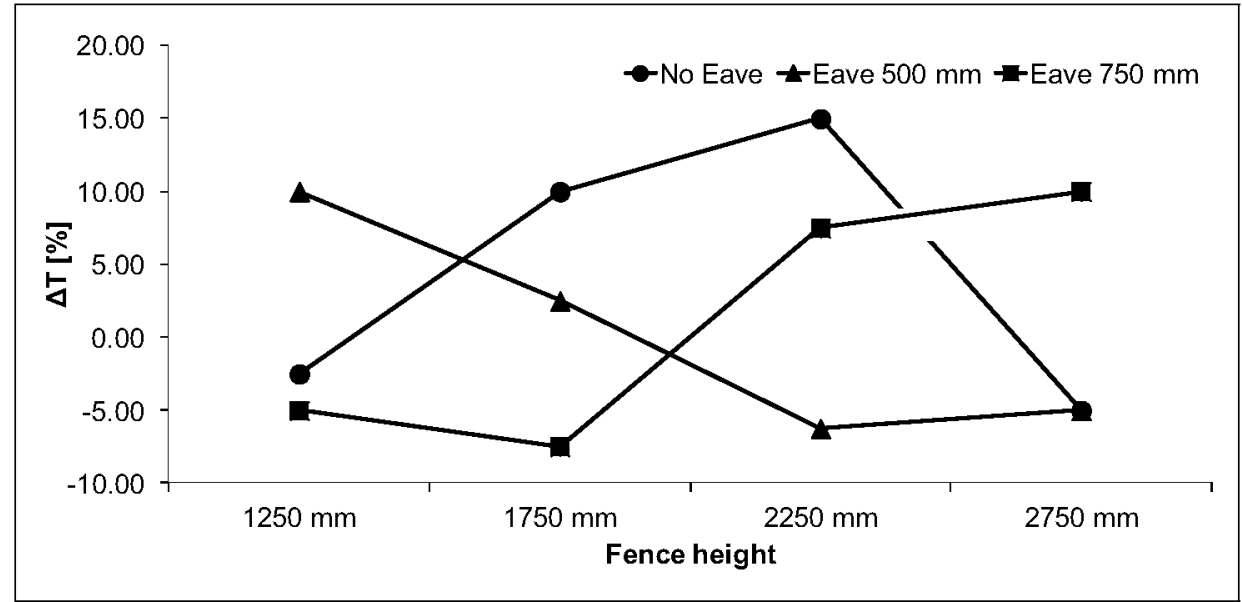

Figure 6. Variation of the time period of the coefficients signals in terms of percentage. The reference configuration is the 'No Fence' scenario. Calculations carried out at $R e \approx 12 \times 10^{6}$.

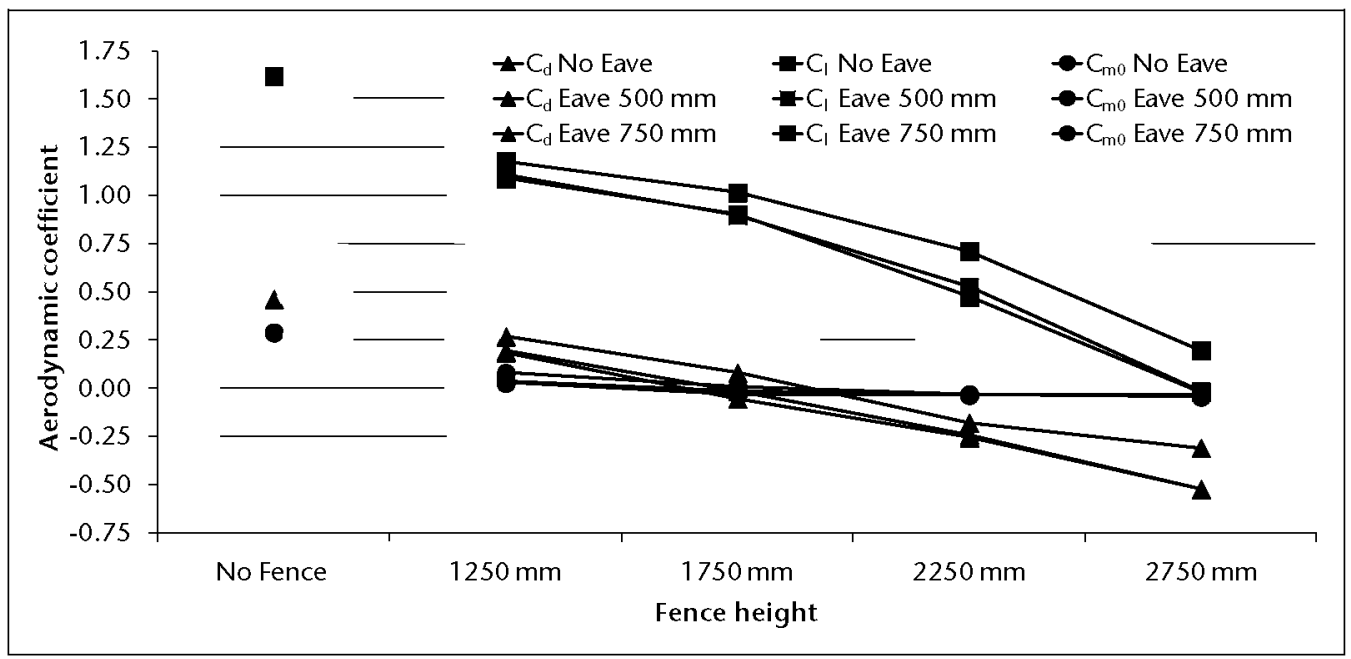

Figure 7. Two-dimensional aerodynamic coefficients of the vehicle $C_{d}, C_{b}, C_{m o}$ calculated at $\operatorname{Re} \approx 12 \times 10^{6}$.

the measured and computed coefficients is larger in the cases of fences with a height of 2250 and $2750 \mathrm{~mm}$. The CFD calculations also match the effect of increasing the value of the parameter $l_{\mathrm{e}}$ from 500 to $750 \mathrm{~mm}$, which appears to be low in both wind tunnel experiments and computed scenarios. Therefore, CFD is a suitable tool for performing a parametric study on fence design and analysing how the air flow is affected by it.

\section{Simulations reproducing strong wind conditions $\left(\operatorname{Re} \approx 12 \times 10^{6}\right)$}

Study of the time signals: Characteristics and influence of fences. In spite of having stationary inlet conditions, the problem under study does not have a stationary solution because of the vortex shedding phenomenon that appears at the leeward side of the bridge (see Figure 5). The flow develops until it reaches a periodic state. Vortices are influenced somewhat by the design of the fence and so they determine the aerodynamic characteristics of the bridge and the vehicle.

Throughout this section the unsteadiness of the flow is discussed in terms of the time period and the peak-to-peak amplitude of the signals. The latter refers to the difference between the highest and lowest values of the signals in the measured time. The average value of the signals, which is the aerodynamic coefficient, will be discussed in the next section.

In the cases of the drag and lift force coefficients, the peak-to-peak amplitudes have an order of $10^{-2}$ in all the considered scenarios, whereas the order of the roll moment coefficient is $10^{-3}$. In all fence configurations, the highest peak-to-peak amplitude values are observed for the drag force coefficient, and the lowest for the roll moment coefficient. The peak-to-peak amplitude of the lift coefficient is somewhere in the middle of the other two coefficients, as shown in Figure 5. In terms of percentage, the peak-to-peak 


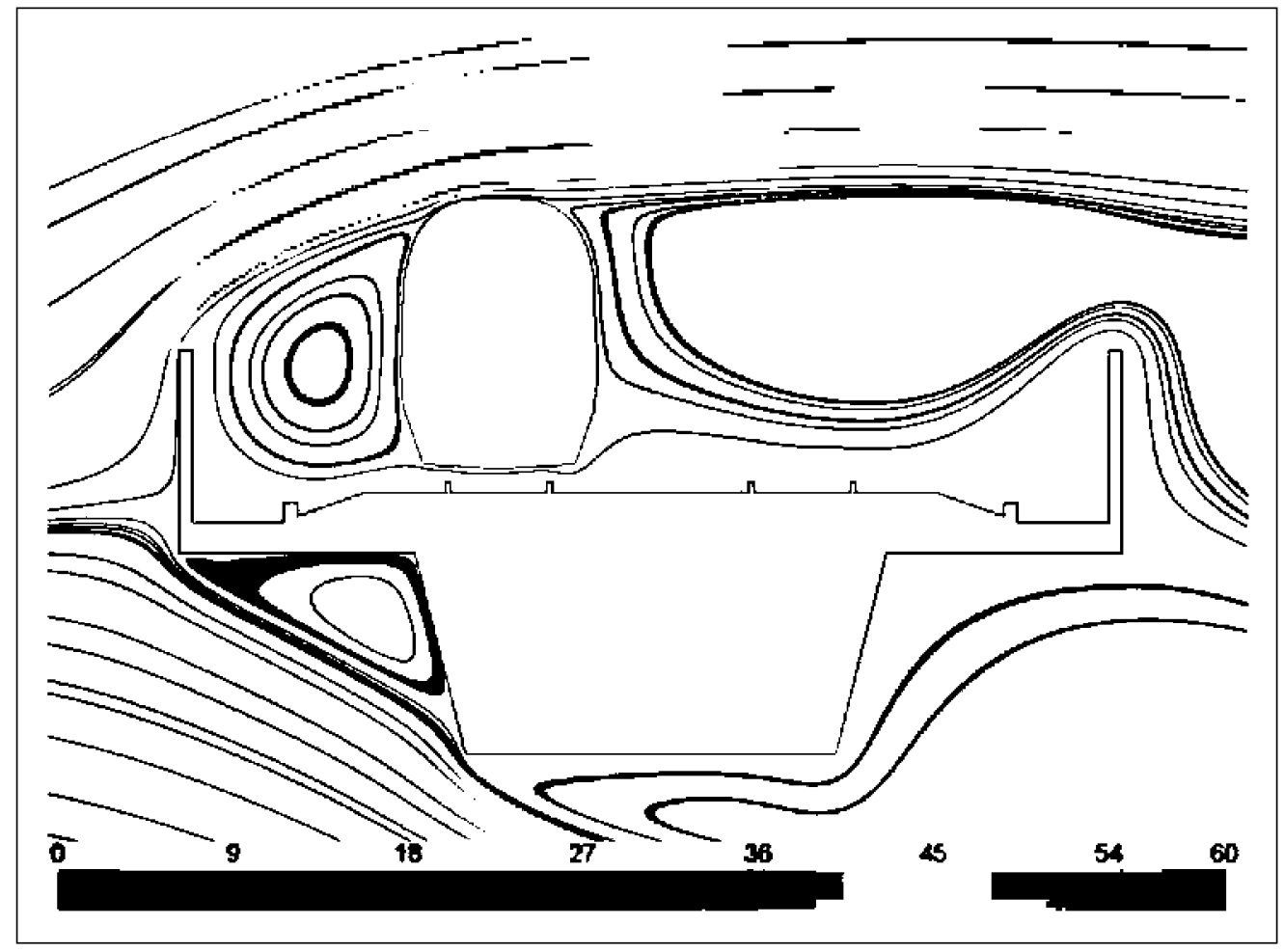

Figure 8. Streamlines coloured with velocity $(\mathrm{m} / \mathrm{s})$ when $R e \approx 12 \times 10^{6}$. Fence $h_{\mathrm{f}}=2250 \mathrm{~mm}$ without eave.

amplitude of the drag coefficient represents around $25 \%$ of the average value, $15 \%$ is the variation of the roll moment coefficient and 5\% corresponds to the lift coefficient. However, it should be taken into account that $C_{m 0}$ has near-zero values and thus small variations provide relatively big values of variation in percentage terms. If modifications to the parapet design are considered, the peak-to-peak amplitude changes without any clear trend.

Time period is also important since it gives information regarding the frequency of the vortex shedding phenomenon. No substantial changes between the periods of the coefficients are observed, so Figure 6 shows how the different fence designs influence the period of the cycles through $C_{l}$. The variation in percentage for the different configurations was calculated by taking as a reference the scenario in which the vehicle is unsheltered, in which case the time period $(T)$ of the lift coefficient is $1.6 \mathrm{~s}(S t \approx 1.1)$. Positive values mean that the period is longer compared with the reference case and negative values indicate shorter periods. In view of the results shown in Figure 6, there is no clear trend that relates the variation of $h_{\mathrm{f}}$ and $l_{\mathrm{e}}$ with the time period. Although $h_{\mathrm{f}}$ and $l_{\mathrm{e}}$ modify the period of the signals, such variations are small. The highest variation is observed in the configuration of a $2250 \mathrm{~mm}$ fence without an eave, where the difference is $15 \%$, which is equivalent to an increase of $0.24 \mathrm{~s}$ over the reference case. If the difference between the maximum and the minimum is calculated, the result is a variation of $22.5 \%$, which involves a variation of $0.4 \mathrm{~s}$.
Effects of the fence design on the aerodynamic coefficients of the vehicle and the flow. Considering the same fence configurations as used in the experimental tests, Figure 7 shows the coefficients of vehicle aerodynamics under strong wind conditions. The values of the aerodynamic coefficients decrease as fence height increases; leading to higher reductions when the fences are built with an eave on the top. Fences have a bigger impact on $C_{l}$ than on $C_{d}$, where the decrease of the lift coefficient value is higher in comparison with the drag or roll coefficients. In fact, the $C_{m 0}$ decreases to zero when the smallest fence is considered $\left(h_{\mathrm{f}}=1250 \mathrm{~mm}\right)$ and its value remains constant despite changes in fence design.

In the scenarios where the fences has no eaves, $C_{d}$ drops from around 0.4 at the maximum (no fence scenario) to -0.4 at the minimum (fence where $h_{\mathrm{f}}=2750 \mathrm{~mm}$ ), as shown in Figure $7 . C_{d}$ has a negative value with fences whose height is greater than $1750 \mathrm{~mm}$ and have an eave as well as with fences whose height is greater than $2250 \mathrm{~mm}$ and do not have an eave. The obtained numerical values of $C_{d}$ suggest that fences can reduce the drag force; however, at the same time they are able to produce suction forces (forces due to negative pressure values) as large as drag forces. What happens with the drag force is that on the windward side, fences reduce the pressure, which decreases as the fence height increases. This happens because a vortex between the fence and the windward side of the vehicle is observed to appear in the configurations where $h_{\mathrm{f}}=2250$ and $2750 \mathrm{~mm}$ (see Figure 8). This vortex accelerates the flow, 


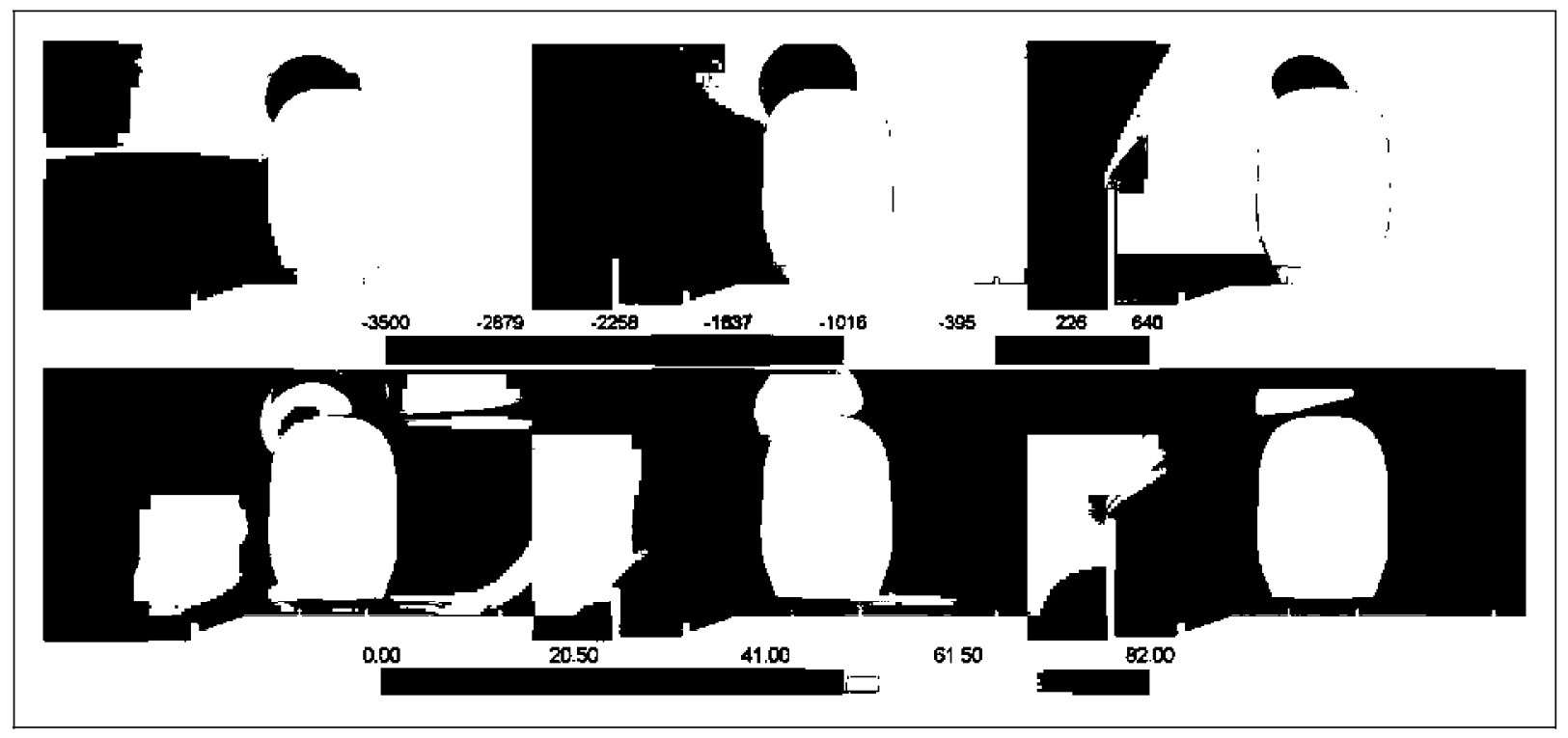

Figure 9. Contours of mean pressure ( $\mathrm{Pa}$ ) (top) and mean velocity $(\mathrm{m} / \mathrm{s})$ (bottom) when $R \mathrm{e} \approx 12 \times 10^{6}$. No fence (left), $h_{\mathrm{f}}=1250 \mathrm{~mm}$ (centre), $h_{\mathrm{f}}=2750 \mathrm{~mm}$ (right).

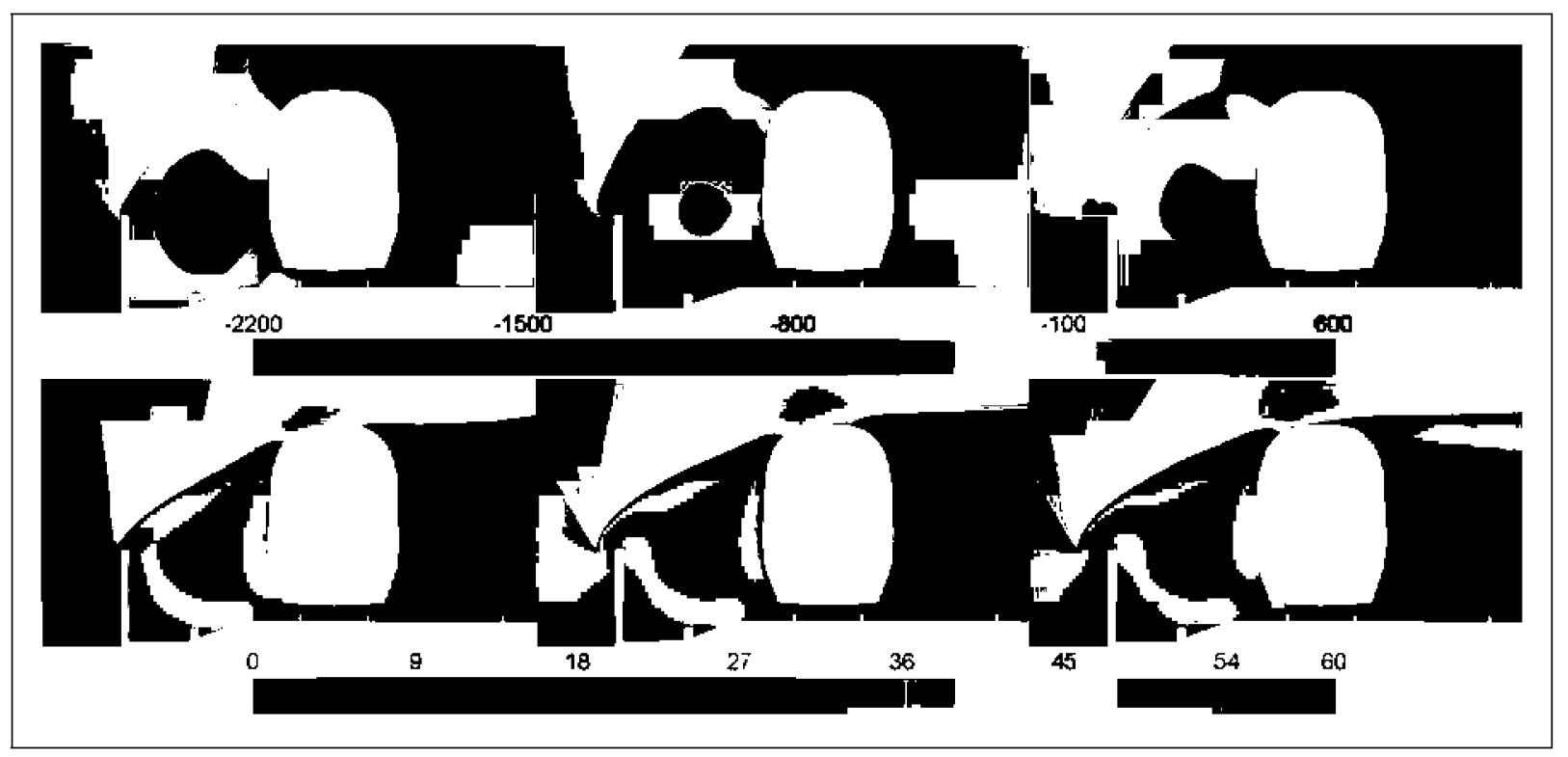

Figure 10. Contours of mean pressure $(\mathrm{Pa})(\mathrm{top})$ and mean velocity $(\mathrm{m} / \mathrm{s})$ (bottom) when $R \mathrm{e} \approx 12 \times 10^{6}$. Fence $h_{\mathrm{f}}=2250 \mathrm{~mm}$. No eave (left) $l_{\mathrm{e}}=500 \mathrm{~mm}$ (centre) $l_{\mathrm{e}}=750 \mathrm{~mm}$ (right).

contributing to a decrease in pressure at the windward side. At the leeward side, the pressure value is almost constant (see Figure 9) because a low-speed zone occurs when the vortex appears at the windward side. All these phenomena work together to decrease the pressure value and produce the same pressure difference between the windward and leeward sides as for the case of the vehicle being on the bridge without any fence

As previously stated, the variation in $C_{l}$ is higher compared with that for $C_{d}$ : whereas $C_{d}$ changed from 0.46 to $-0.31, C_{l}$ went from 1.6 to 0.4 . Thus, fences have a greater effect on the difference in pressure between the top and bottom of the vehicle than on the sides (see Figure 9). As the fence height increases suction at the top decreases, and the pressure also reduces at the bottom, though at a lower rate; as a result, the pressure difference is significantly reduced. When a fence is added, the flow deviates and the interaction between the main air stream and the windward side of the train gradually reduces when the fence height is increased. As long as the fence is low, the flow reaches the carbody of the vehicle near its bottom, which produces an acceleration of the flow to pass over the coach. This acceleration is high enough to accelerate the flow to $78 \mathrm{~m} / \mathrm{s}$ for the configuration where the vehicle is unsheltered. An increase in the fence height helps the flow to pass over the 


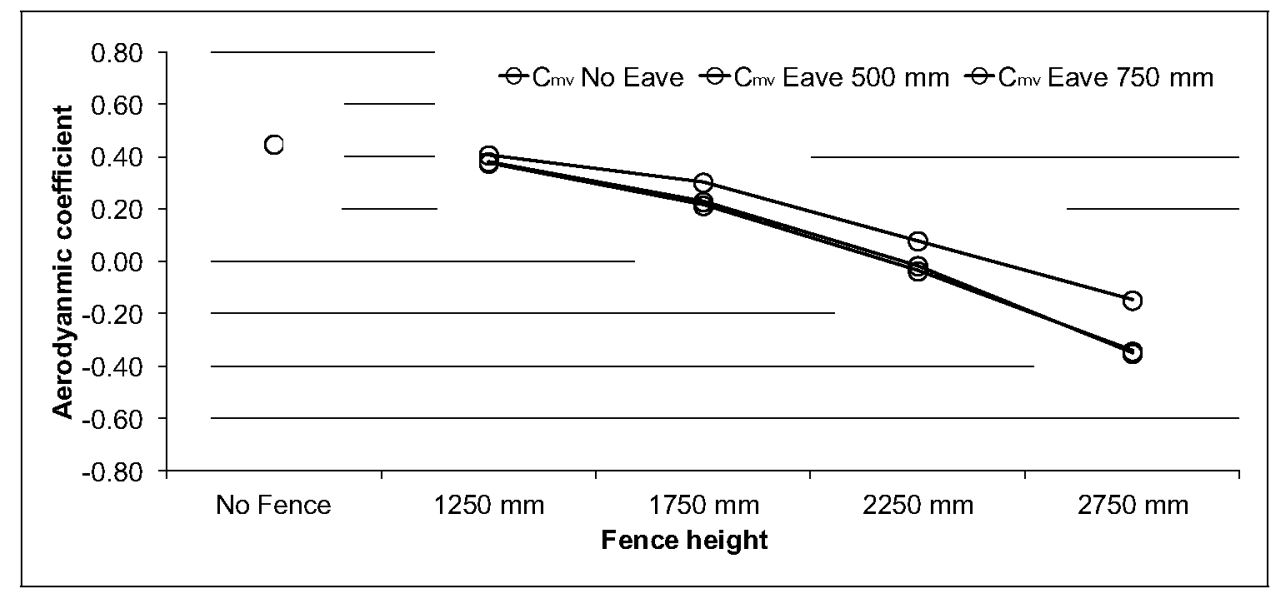

Figure I I. Two-dimensional aerodynamic coefficient of moment around the leeward rail $\left(C_{m v}\right)$ of the vehicle calculated at $\operatorname{Re} \approx 12 \times 10^{6}$.

coach; as a result, the flow needs to accelerate less, which reduces the suction forces at the top of the vehicle. These suction forces are verified to be the main cause of the high values of $C_{l}$, because the forces produces a large difference in pressure between the top and bottom of the carbody; as long as fences are able to change the flow direction over the carbody, $C_{l}$ decreases.

Pressure at the bottom of the vehicle also needs to be taken into account to fully understand the behaviour of the lift coefficient. An airstream goes under the vehicle, which accelerates due to the reduction of the cross-sectional area, modifying the flow at the leeward side of the vehicle (Figure 8). The flow of the airstream is affected by fences; as they increase in height, the flow slows down; thus, the pressure at the bottom increases slightly. In addition, the flow under the coach is influenced by the suction that appears at the windward side when the fence height is $h_{\mathrm{f}}=2250$ and $2750 \mathrm{~mm}$. In such cases the direction of the airstream goes from leeward to windward (see Figure 9), feeding the vortex that appears at the windward side. However, in spite of the airstream flowing in the opposite direction, the pressure under the coach does not change.

It was previously stated that adding an eave to the fences reduces the value of the loads acting on the vehicle and thus the coefficients (see Figure 7). Nevertheless, the simulations show that despite increasing $l_{\mathrm{e}}$ from 500 to $750 \mathrm{~mm}$, the coefficients change their value only slightly; therefore, the parameter $l_{\mathrm{e}}$ does not seem to be very important, as the variation is almost zero. It should be noted that adding eaves has a greater effect on the lift force than on the drag and roll moment coefficients. In addition, $C_{l}$ reaches zero in the case where the fence has a height $h_{\mathrm{f}}=2750 \mathrm{~mm}$ and an eave.

Figure 10 shows the pressure and velocity around the vehicle in the scenario where the fence height is $h_{\mathrm{f}}=2250 \mathrm{~mm}$. It compares the outcome of adding an eave and then the importance of the parameter $l_{\mathrm{e}}$.
Simulations show that the inclusion of eaves results in an additional decrease of the suction at the top of the vehicle, which decreases by around 0.3 (see Figure 7) the value of $C_{l}$ for a fence height of $h_{\mathrm{f}}=1750 \mathrm{~mm}$ and above (see Figure 7). What the eave does is to produce an additional deviation of the flow so the main flow tends to gain distance with the coach as the value of $l_{\mathrm{e}}$ is higher, causing two opposite effects. On the one hand, the flow is reoriented by the eave, so it needs even less acceleration to pass over the carbody; that makes the suction forces decrease, so $C_{I}$ reduces as well. On the other hand, an effect that has been predicted by other authors appears, which is that the flow has bigger values for the velocity at higher positions. This increases the turbulence at positions near the catenary, which may result in the aero-elastic phenomenon of galloping of the cable. ${ }^{7}$ Nevertheless, increasing $l_{\mathrm{e}}$ from 500 to $750 \mathrm{~mm}$ does not modify the direction of the flow enough to change the pressure field, so the aerodynamic coefficients decreases slightly.

Looking at Figure 10 and the difference in pressure between the windward and leeward sides of the vehicle in the cases where the fences have an eave, it is clear that pressure decreases slightly at the windward side and the pressure value at the leeward side remains constant. This results in a small variation in $C_{d}$ for both the cases of a fence with an eave and a fence without an eave, and the same thing happens between $l_{\mathrm{e}}=500 \mathrm{~mm}$ and $l_{\mathrm{e}}=750 \mathrm{~mm}$.

Figure 11 shows the calculation of the moment coefficient around the leeward rail $\left(C_{m v}\right)$. It shows that the value of $C_{m v}$ decreases from 0.45 to 0.41 when $h_{\mathrm{f}}=1250 \mathrm{~mm}$, regardless of whether or not the fence has eaves; therefore, fences without eaves can only be considered efficient with heights of $h_{\mathrm{f}}=1750 \mathrm{~mm}$ and above. With this configuration, $C_{m v}$ decreases to -0.17 with $h_{\mathrm{f}}=2750 \mathrm{~mm}$. Fences built with eaves manage to decrease $C_{m v}$ from around 0.4 to -0.34 , mainly from the effect of the suction forces which were explained earlier, turning 


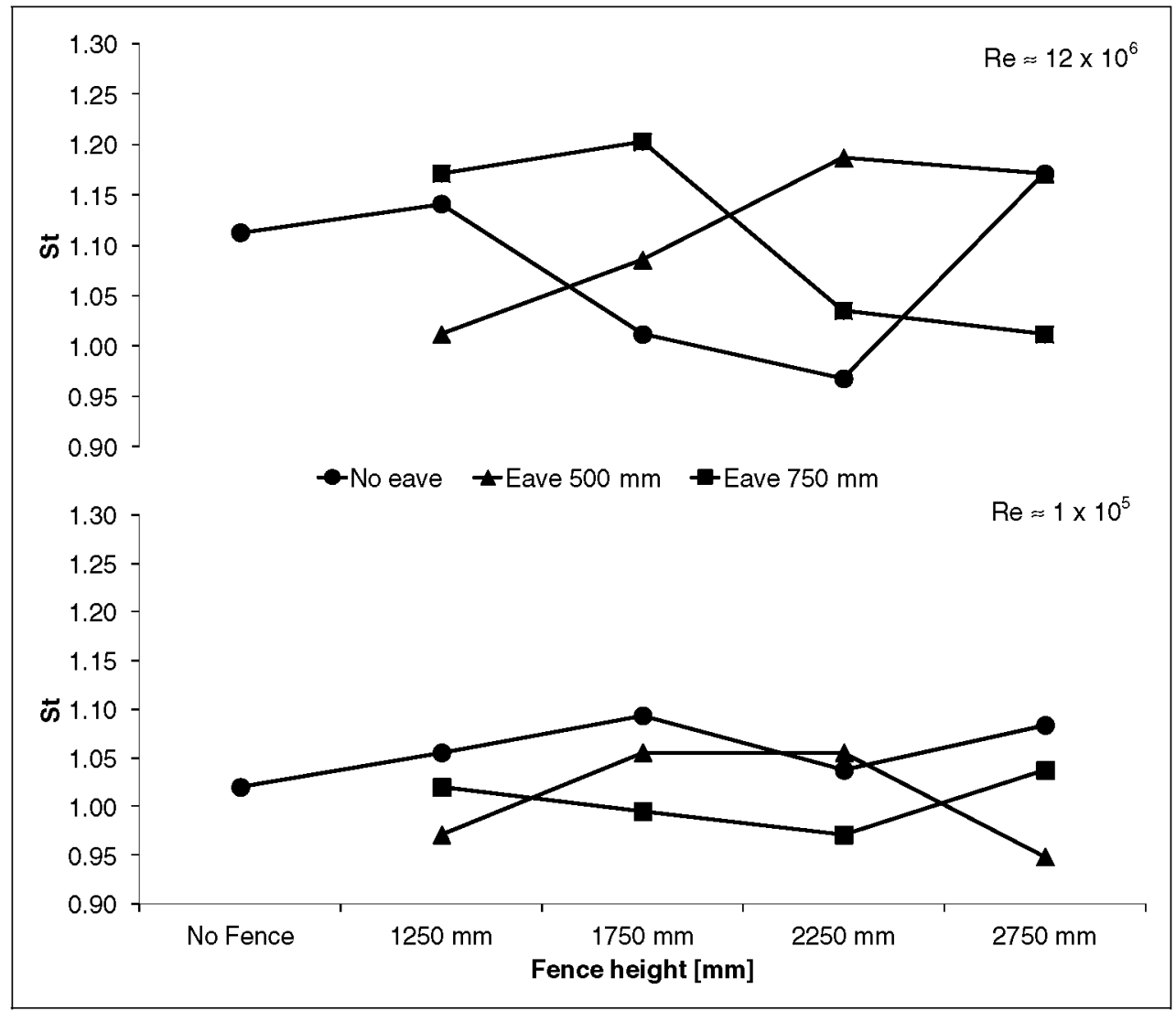

Figure 12. Strouhal number calculated in all the scenarios and for the two Re values.

$C_{d}$ into negative values. In safety terms, this means an overturning moment as big as when the vehicle was unsheltered but in the opposite direction. However, this result appears to be influenced by the twodimensional space in which this study was performed. It is expected that high fences may produce suction forces but not as high as the ones predicted in these simulations. A three-dimensional study of this configuration should confirm and quantify the result.

The effect on $C_{m v}$ of adding an eave increases with fence height. Eaves do not produce any additional decrease in the coefficient for the case of the lowest fence; the efficiency of eaves grows as the height is increased and eaves reduce the value of $C_{m v}$ by an additional 0.2 when $h_{\mathrm{f}}=2750 \mathrm{~mm}$. The final conclusion with respect to eaves is that an increase in eave length from 500 to $750 \mathrm{~mm}$ does not improve safety conditions, since $C_{m v}$ remains constant.

\section{The dependency of the aerodynamic coefficients on the Reynolds number}

The two-dimensional section of the vehicle plus the bridge under consideration in this study could also be classified as a more-or-less bluff body inside an airstream. In such cases, the Reynolds number is of vital importance since it can have a major influence on certain flow parameters. In light of the CFD results, this section studies what these magnitudes are, and in the case they are affected by $R e$, analyses whether the change is considerable.

Effects on the time signals: Characteristics and influence of fences. The unsteadiness of the flow was discussed thoroughly in the section 'Study of the time signals: characteristics and influence of fences', in particular the time period and the peak-to-peak amplitude of the time signals. In this section, the influence of $R e$ on the frequency of the vortex shedding phenomenon is studied in terms of the time period $(T)$ and the Strouhal number $(S t)$. The effect of $R e$ on the peak-to-peak amplitude is also investigated.

$R e$ has a strong influence on the period of the coefficients' signal. It was mentioned in the section 'Study of the time signals: characteristics and influence of fences' that for simulations carried out with $R e \approx 12 \times 10^{6}$ the period is around $1.5 \mathrm{~s}$. If the same scenarios are simulated with $R e \approx 1 \times 10^{5}$, the period oscillates in the interval $225<T<250 \mathrm{~s}$, where the time period of the bridge without a fence is $238 \mathrm{~s}$. If variations with respect to the case of an unsheltered vehicle are calculated in the simulations at $R e \approx 1 \times 10^{5}$, by applying the methodology that was used for Figure 6, the maximum variation appears for the case of fence whose parameters are $h_{\mathrm{f}}=2750 \mathrm{~mm}$ and $l_{\mathrm{e}}=500 \mathrm{~mm}$. This variation is $7.5 \%$ and corresponds to $20 \mathrm{~s}$ whereas the maximum difference at $R e \approx 12 \times 10^{6}$ is approximately $15 \%$. Furthermore, 


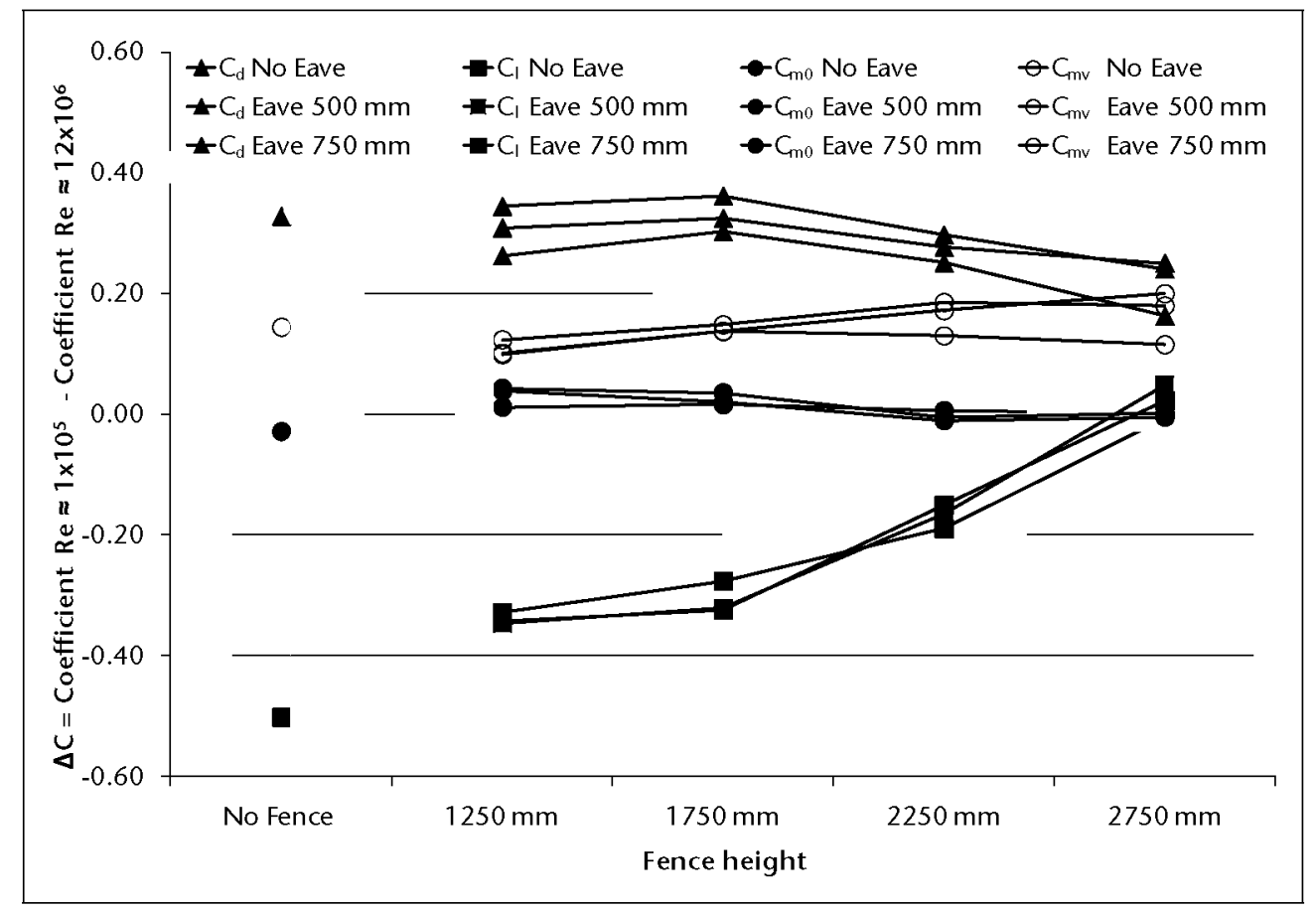

Figure 13. Reynolds dependency of the aerodynamic coefficients $C_{d}, C_{b}, C_{m o}$. Difference between tests at $\operatorname{Re}=91,900$ and $R e \approx 12 \times 10^{6}$.

the difference between the maximum and minimum values of the period is $25 \%$ at $R e \approx 12 \times 10^{6}$ (see Figure 6), decreasing to $15 \%$ at $R e \approx 1 \times 10^{5}$.

Figure 12 shows the result of calculating $S t$ in all the cases considered in the present study arranged by $R e$. Analysing the results at each $R e$ separately, it can be concluded that no clear trend exists between the fence design parameters and the Strouhal number. Furthermore, $S t$ behaviour changes when Re increases, which makes it impossible to analyse the effect of $R e$ on $S t$. The graph shows that $S t$ changes slightly between the two $R e$ values. For simulations at a low Reynolds number $\left(R e \approx 1 \times 10^{5}\right)$ the $S t$ is always between 0.95 and 1.10 , whereas for simulations at $R e \approx 12 \times 10^{6}$, it varies between 0.95 and 1.2. The small changes in $S t$ whenever $R e, h_{\mathrm{f}}$ or $l_{\mathrm{e}}$ are changed makes it impossible to determine what changes happen in the flow to cause the variation in $S t$. Lastly, by modifying a parameter of the fence design $\left(h_{\mathrm{f}}\right.$ or $l_{\mathrm{e}}$ ), changes are smooth at $R e \approx 12 \times 10^{5}$, whereas at $R e \approx 12 \times 10^{6}$ they are not smooth.

Regarding peak-to-peak amplitude, it is possible to add that for the three aerodynamic coefficients, their order at $R e \approx 12 \times 10^{5}$ is the same as the ones presented in the section 'Study of the time signals: characteristics and influence of fences'. At low Re, peak-to-peak amplitude only represents $5 \%$ of the average value of $C_{d}, C_{l}$ and $C_{m 0}$, whereas at $R e \approx 12 \times 10^{6}$ the values are 25,5 and $15 \%$, respectively.

Effects of the fence design on the aerodynamic coefficients of the vehicle and the flow. It has been shown that $R e$ has an influence on the period and the peak-to-peak amplitude of the coefficients' time signals. In this section, whether carrying out tests at a lower $R e$ value alters the coefficients average value is assessed.

Figure 13 shows the influence of $R e$ on the numerical results for $C_{d}, C_{l}, C_{m 0}$ and $C_{m v}$. The analysis was performed by defining $\Delta C$ as the difference between the coefficient's average value at $R e \approx 1 \times 10^{5}$ and at $R e \approx 12 \times 10^{6}$; in this way, a positive value means that coefficients at the lower $R e$ are higher. Thus, at $R e \approx 1 \times 10^{5}, C_{d}$ has higher values and $C_{l}$ has smaller values. According to these results, $C_{m 0}$ can be considered to be independent of $R e$ since the value changes by less than 0.04 . The highest dependency on $R e$ is observed in the configuration where there is no fence on the bridge, whereas the minimum dependency is seen in the cases where the fence has its highest value, i.e. $h_{\mathrm{f}}=2750 \mathrm{~mm}$. In analysing the coefficients' trends and values, it is concluded that each one behaves differently. The drag force coefficient shows a variation in the range $0.16-0.36$. The lift force coefficient is highly dependent on $R e$. When the vehicle stands on the bridge without being protected by a fence, $\Delta C_{l}$ reaches the maximum value of all coefficients, a difference of -0.5 . If fences are added to the scenario, the coefficient tends to be more independent of $R e$, the difference is almost zero when $h_{\mathrm{f}}=2750 \mathrm{~mm}$.

The variation in $C_{m v}$ is small, oscillating between 0.1 and 0.2 . It reaches its maximum value when $h_{\mathrm{f}}=2750 \mathrm{~mm}$ and there is no eave, and the minimum appears in the case where $h_{\mathrm{f}}=1250 \mathrm{~mm}$ and there is no eave. In general terms, $\Delta C_{m v}$ tends to have the 
value of $\Delta C_{d}$, getting closer to it as the fence height increases since $\Delta C_{l}$ also approaches zero. In calculating $C_{m v}$, the drag force coefficient prevails over $C_{l}$ and that is reflected here, too. $C_{m 0}$ also has an influence on $C_{m v}$ though it is shown in Figure 13 that $R e$ has no effect on it. The fact that the value of $\Delta C_{m v}$ is given mainly by the variation of $C_{d}$ is easy to check in the 'No Fence' case and in the configuration where $h_{\mathrm{f}}=2750 \mathrm{~mm}$. When the vehicle is unprotected, the variation in $C_{l}$ reaches its maximum value; however, $\Delta C_{m v}$ is clearly close to $\Delta C_{d}$, which is an indication of the effect of the drag force coefficient on $C_{m v}$. In addition, $\Delta C_{m v}$ almost has the same value as $\Delta C_{d}$ when $h_{\mathrm{f}}=2750 \mathrm{~mm}$ since in this case the variation in the lift and roll moment coefficients is almost zero, and thus only the drag force coefficient sets the value of $\Delta C_{m v}$.

Eaves have a small influence on the conclusions presented in this section. Adding an eave results in a small increase in the dependence of $C_{d}$ on $R e$ in all the fence designs. The influence of $R e$ relies on the fence height in the case of $C_{l}$. For the small fences, $h_{\mathrm{f}}=1250$ and $1750 \mathrm{~mm}$, adding an eave makes the coefficient more sensitive to Re but the opposite happens for the other two fence heights. Lastly, $\Delta C_{m v}$ increases from 0.1 to approximately 0.2 in the cases where $h_{\mathrm{f}}=2250$ and $2750 \mathrm{~mm}$. What does not seem to be relevant in this matter is eave length since the variation in $\Delta C$ for every fence design is near zero in spite of having increased $l_{\mathrm{e}}$ from 500 to $750 \mathrm{~mm}$.

\section{Conclusions}

This paper studied the relative effectiveness of windbreaks built on a bridge to protect trains. The problem did not have a stationary solution, thus it was studied in the time domain. A vortex shedding phenomenon appeared despite having stationary inlet conditions. It was shown that a CFD approach is able to reproduce the overall trends of the aerodynamic coefficients with fence height and the presence of eaves in agreement with the experiments carried out in a wind tunnel. Moreover, it was shown that a CFD model that represents a vehicle on a bridge is a useful tool for carrying out a parametric study as an alternative to performing experimental tests.

Real wind conditions were simulated, which showed that the fence design modifies the flow and thus the efficiency of the protection. When fence height increases, wind loads are reduced but fences must be high enough to provide the protection needed to safeguard the train's running. Results showed that fences are able to modify the flow in such a way that suction forces that are as large as drag forces can appear when the fence height is large. This effect is enhanced with the inclusion of an eave. The CFD model showed that fences had a more significant influence on lift force than on drag force, and the roll moment approached zero when any fence was installed. The presence of an eave slightly increased the shielding effect of fences, and it had a higher impact on the lift force coefficient than on the others. Furthermore, simulations and experiments showed that there is no difference between the scenarios whether the eave length was 500 or $750 \mathrm{~mm}$.

With the cross-sectional area of the vehicle plus the bridge being a more-or-less bluff body, the aerodynamic properties changed with $R e$. The vehicle coefficients followed the same trend with the two Reynolds numbers used in the simulations; nevertheless, the numerical values changed sufficiently to consider that the simulations carried on at $R e \approx 1 \times 10^{5}$ are not enough to decide which fence design is the best in conjunction with the information from the wind tunnels tests. The $C_{d}$ value decreased around an average of 0.31 when simulations were performed at $R e \approx 12 \times 10^{6} . C_{m 0}$ was independent of $R e$, and the behaviour of $C_{l}$ depended on the fence design. Changes in $R e$ also slightly affected $S t$ since the result of the comparison gave relatively similar values in spite of the increased $R e$ value. Nevertheless, when the frequency of the coefficients was studied in terms of the period of the coefficients' signals, their value decreased from around $250 \mathrm{~s}$ when simulations were carried out at $R e \approx 1 \times 10^{5}$ to $1.5 \mathrm{~s}$ when $R e$ was $12 \times 10^{6}$.

This parametric study using two-dimensional models needs to be complemented by specific threedimensional calculations in order to provide the full three-dimensional flow and consequently a good estimation of actual wind forces with different fence characteristics. The computational effort of such models is high so that parametric studies are generally not affordable.

\section{Funding}

This research received no specific grant from any funding agency in the public, commercial or not-for-profit sectors.

\section{References}

1. Schulte-Werning B, Grégorie R, Malfatti A and Matschke G. (eds). TRANSAERO-A European initiative on transient aerodynamics for railway system optimisation. Springer, 2002, p.377.

2. Schewe G and Larsen A. Reynolds number effects in the flow around a bluff bridge deck cross section. J. Wind Engng Ind Aerodyn 1998; 74-76: 829-838.

3. Schewe G. Reynolds-number effects in flow around more-or-less bluff bodies. $J$ Wind Engng Ind Aerodyn 2001; 89(14-15): 1267-1289.

4. Larose GL and D'Auteuil A. On the Reynolds number sensitivity of the aerodynamics of bluff bodies with sharp edges. J. Wind Engng Ind Aerodyn 2006; 94(5): 365-376.

5. Matsuda K. An investigation of Reynolds number effects on the steady and unsteady aerodynamic forces on a 1:10 scale bridge deck section model. $J$ Wind Engng Ind Aerodyn 2001; 89(7-8): 619-632. 
6. Mannini C, Soda A, VoB R and Schewe G. Unsteady RANS simulations of flow around a bridge section. $J$ Wind Engng Ind Aerodyn 2010; 98: 742-753.

7. Avila-Sanchez S, Meseguer $\mathbf{J}$ and Lopez-Garcia $\mathrm{O}$. Turbulence intensity on catenary contact wires due to parapets placed on a double track bridge. $J$ Wind Engng Ind Aerodyn 2010; 98(10-11): 504-511.

8. Bettle J, Holloway AGL and Venart JES. A computational study of the aerodynamic forces acting on a tractor-trailer vehicle on a bridge in cross-wind. $J$ Wind Engng Ind Aerodyn 2003; 91(5): 573-592.

9. Diedrichs B. Aerodynamic crosswind stability of a regional train model. Proc IMechE, Part F: $J$ Rail Rapid Transit 2010; 224(6): 580-591.

10. Diedrichs B. On computational fluid dynamics modelling of crosswind effects for high-speed rolling stock. Proc IMechE, Part F: J Rail Rapid Transit 2003; 217(3): 203-226.

11. Cheli F, Giappino S, Schito P, et al. Experimental analysis on the effect of windbreak fences on railway vehicles. In: The International Association for Vehicle System Dynamics conference (ed. Manchester Metropolitan University), Manchester, UK, 14-19 August 2011. pp.1-6.

12. Barcala $\mathbf{M}$ and Meseguer $\mathbf{J}$. An experimental study of the influence of parapets on the aerodynamic loads under cross wind on a two-dimensional model of a railway vehicle on a bridge. Proc IMechE, Part F: J. Rail Rapid Transit 2007; 221(4): 487-494.

13. Sanz J de D, Brunel JA and Crespo P. Adverse aerodynamics effects of a high speed train under the influence of cross winds, analysis and optimization of wind fence. In: The International Association for Vehicle System Dynamics conference, Manchester, UK, 2011, pp.1-5.

14. ANSYS Inc. ANSYS FLUENT reference guide. ANSYS, 2010.

15. Issa RI. Solution of implicitly discretized fluid flow equations by operator splitting. J Comput Phys 1986; 62(1): 40-65.

16. Mathur SR and Murthy JY. A pressure-based method for unstructured meshes. Numer Heat Transfer 1997; 31(2): 195-215.

17. EN 1991 1-4 Eurocode 1: 2005. Eurcode 1: 2005. Actions on structures - part 1-4: general actions - wind actions.

18. Suzuki M, Tanemoto K and Maeda T. Aerodynamic characteristics of train/vehicles under cross winds. $J$ Wind Engng Ind Aerodyn 2003; 91: 209-218.

19. Maskell EC. A Theory of the blockage effects on bluff bodies and stalled wings in a closed wind tunnel. ARC R\&M. 1965; (3400).

20. Barlow JB, Rae WH and Pope A. Low-speed wind tunnel testing. New York, NY: John Wiley \& Sons, 1999. 